\title{
Beating the sign problem in finite density lattice QCD
}

\section{Zn Collaboration}

\section{Ryutaro Fukuda}

Department of Physics, The University of Tokyo, Tokyo 113-0033, Japan

Institute für Theoretische Physik, ETH Zürich, CH-8093 Zürich, Switzerland,

\section{Atsushi Nakamura*}

RCNP, Osaka University, Osaka567-0047, Japan

Nishina Center, RIKEN, Wako, Saitama 351-0198, Japan

School of Biomedicine, Far Eastern Federal University, Vladivostok 690950, Russia

E-mail: atsushi@renp.osaka-u.ac.jp

\section{Shotaro Oka}

Institute of Theoretical Physics, Rikkyo University Toshima-ku, Tokyo 171-8501, Japan

\section{Shuntaro Sakai}

Department of Physics, Kyoto University, Kyoto 606-8502, Japan

\section{Asobu Suzuki and Yusuke Taniguchi}

Graduate School of Pure and Applied Sciences, University of Tsukuba, Ibaraki 305-8571, Japan

\begin{abstract}
We report our recent project to study the QCD phase diagram by the canonical approach (CA), which is expected to solve the sign problem. First we briefly describe the sign problem and several approaches to fight against it. Then we argue that the CA may be a break-through if we combine it with the multiple precision calculation. We study the method using the hopping parameter expansion (HPE), in order to investigate how the CA works. We show explicitly how we calculate the winding number in HPE and obtain the fugacity expansion whose coefficients are the canonical partition functions. Finally we discuss dark sides of the current CA, which should be beaten for the method to become a real tool of QCD phase explorers.
\end{abstract}

The 33rd International Symposium on Lattice Field Theory

14 - 18 July 2015

Kobe International Conference Center, Kobe, Japan

\footnotetext{
${ }^{*}$ Speaker.
} 


\section{Introduction - "Sign problem"}

The objective of our project is to clarify the QCD phase structure, and especially to determine the QCD phase transition line. A well-know obstacle of this goal is "sign problem", that is caused by a complex determinant appearing in the path integral,

$$
Z(\mu, T)=\operatorname{Tr} e^{-(H-\mu \hat{N}) / T}=\int \mathscr{D} U(\operatorname{det} \Delta(\mu))^{\mathrm{N}_{\mathrm{f}}} \exp \left(-S_{G}\right),
$$

where $\mu$ and $T$ are the chemical potential and temperature, and $S_{G}$ and $\Delta$ stand for the gluon action and the fermion (quark) determinant with the number of flavors $N_{f}$. We consider $N_{f}=2$ case in this report.

The problem comes due to the relation,

$$
(\operatorname{det} \Delta(\mu))^{*}=\operatorname{det} \Delta\left(-\mu^{*}\right)
$$

which guarantees that $\operatorname{det} \Delta(\mu)$ is real at $\mu=0$, but not for $\mu>0$. Monte Carlo simulations generate configurations $\{U\}$ in the path integral formula 1.1. with the probability proportional to $(\operatorname{det} \Delta(\mu))^{\mathrm{N}_{\mathrm{f}}} \exp \left(-S_{G}\right)$. Thus when $\operatorname{det} \Delta(\mu)$ is complex, we are in a dither.

There are several cases when $\operatorname{det} \Delta(\mu)$ is real:

i. Color SU(2)

ii. Phase quench or the finite isospin

iii. Pure imaginary chemical potential

iv. Chiral chemical potential

The two color QCD (ii) is a very good test bed for studying theoretical aspect of the finite density theory and have been used since Ref.[1]. But, it is not suitable for predicting the phase transition point quantitatively and for clarifying its nature.

The finite isospin simulations (1ii) [2, 3, 4 ${ }^{1}$ describe a slightly different world from QCD, and they suffers a pion condensation at $\mu=m_{\pi} / 2$.

In this report, we discuss general features of the canonical approach (CA) and a relation between the winding number expansion and the hopping parameter expansion. Physical outcomes, its comparison with the multi-parameter reweighting method, the moments of the baryon number and an analysis of the high precision calculation are discussed by Ref.[5], [6], [7] and [8], respectively.

\section{Several approaches}

Since the quark determinant $\operatorname{det} \Delta(\mu)$ is real when the chemical potential $\mu$ is pure imaginary, i.e., $\mu^{2}<$, one way to calculate $Z(\mu, T)$ in Eq.11.1) is to extrapolate results from $\mu^{2}<0$ to $\mu^{2}>0$ [910]. This is a giant step for the finite density lattice QCD towards beating the sign problem yoke, and yet of course it is limited to small chemical potential values because it is an extrapolation. See Fig.12 in Ref.[11].

\footnotetext{
${ }^{1}$ In Refs. 3] 4 , they call it iso-vector type chemical potential.
} 
Another great step for the finite density lattice QCD was the multi-parameter reweighting method[12], in which observables are calculated as

$$
\begin{aligned}
\langle O\rangle & =\frac{1}{Z(\mu)} \int \mathscr{D} U O \operatorname{det} \Delta(\mu, U) e^{-\beta S_{G}} \\
& =\frac{1}{Z(\mu)} \int \mathscr{D} U O \frac{\operatorname{det} \Delta(\mu, U)}{\operatorname{det} \Delta(\mu=0, U)} e^{-\left(\beta-\beta_{0}\right) S_{G}} \operatorname{det} \Delta(\mu=0, U) e^{-\beta_{0} S_{G}} \\
& =\left\langle O \frac{\operatorname{det} \Delta(\mu, U)}{\operatorname{det} \Delta(\mu=0, U)} e^{-\left(\beta-\beta_{0}\right) S_{G}}\right\rangle_{0} /\left\langle\frac{\operatorname{det} \Delta(\mu, U)}{\operatorname{det} \Delta(\mu=0, U)} e^{-\left(\beta-\beta_{0}\right) S_{G}}\right\rangle_{0}
\end{aligned}
$$

The Monte Carlo update is done at $\mu=0^{2}$, and therefore no sign problem occurs.

At first glance, there is no restriction of the regions of $\mu / T$. But the reweighting factor, $\operatorname{det} \Delta(\mu, U) / \operatorname{det} \Delta(0, U)$ is complex and fluctuate. In Fig 1 we show the scatter plots of $(\operatorname{det} \Delta(\mu, U) /$ $\operatorname{det} \Delta(\mu=0, U))^{2}$, namely the reweighting factor appearing in Eq. 22.1] [13]. We see that they fluctuate in the complex plane, and the fluctuation increases as $\mu$ becomes large, that makes the simulation impossible. See Fig.4 in Ref.[13] for more details.

\section{Canonical approaches}

The grand partition function $Z(\mu, T)$ and the canonical partition functions $Z_{n}$ are related as

$$
\begin{aligned}
Z(\mu, T) & =\operatorname{Tr} e^{-(H-\mu \hat{N}) / T}=\sum_{n=-N_{\max }}^{+N_{\max }}\left\langle n\left|e^{-H / T}\right| n\right\rangle e^{\mu n / T} \\
& =\sum_{n=-N_{\max }}^{+N_{\max }} Z_{n}(T) \xi^{n}
\end{aligned}
$$

where $\xi=\exp (\mu / T)$ is fugacity, and

$$
Z_{n}=\langle n|\exp (-H / T)| n\rangle \text {. }
$$

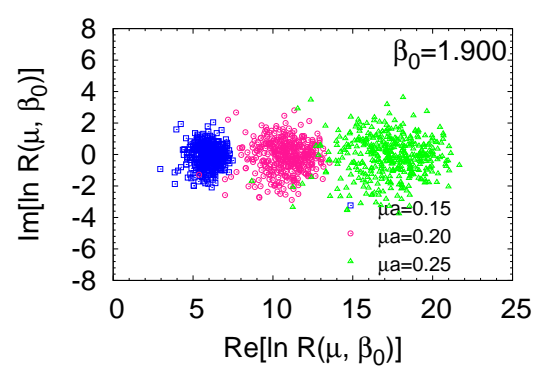

Figure 1: Scatter plots of the fermion reweighting factor, $R=$ $(\operatorname{det} \Delta(\mu, U) / \operatorname{det} \Delta(0, U))^{N_{f}}, \quad$ at $\quad \beta=1.8$ and $1.9[13]$.

Here we assume that the number operator $\hat{N}$ commutes with $H$, that is, $\hat{N}$ is a conserved quantity. $\hat{N}$ can be any conserved number operators, such as baryon, charge and strangeness.

One way to calculate the canonical partition functions, $Z_{n}$, is the Fourier transformation of the grand partition function at pure imaginary chemical potential ${ }^{3}[15]$,

$$
Z_{n}=\int_{-\pi}^{+\pi} \frac{d \theta}{2 \pi} e^{i n \theta} Z\left(\theta \equiv \frac{\mu_{I}}{T}\right)
$$

where $\mu_{I}$ is pure imaginary chemical potential. The grand partition function, $Z$, is evaluated at the pure imaginary chemical potential, and therefore there is no sign problem. This is an appealing idea and several trials have been done [16, 17, 18, 19,20, 21, 22, 23]. But, still we are far from the

\footnotetext{
${ }^{2}$ One can update also at a pure imaginary chemical potential values instead of $\mu=0$.

${ }^{3}$ Another way is to employ the reduction formula 14; In Ref.[13], explicit form of the fugacity expansion from the reduction formula was discussed.
} 
final goal; this is because the canonical partition functions fluctuate in the complex plane during the Monte Carlo update process. We will show the problem later.

If we can calculate the canonical partition functions, $Z_{n}$, then Eq 3.1 allows us to calculate any quantity at the real chemical potential, i.e., the real finite density region.

From Eq. 3.1], we can evaluate also the grand canonical partition function at the complex fugacity plane. Zeros of $Z(\xi)$ are called Lee-Yang zeros 24. In Fig 2 we show a result of LeeYang zero obtained by using this formula.

\subsection{Hopping parameter expansion $\rightarrow$ Winding number expansion}

In order to see whether CA will beat the sign problem or it is an empty dream, we must study its advantage and drawback; especially if we can improve the disadvantage aspects. For this purpose, we investigate a lattice QCD on a small lattice with the hopping parameter expansion.

We start from

$$
\begin{aligned}
& \operatorname{det} \Delta=\exp (\operatorname{Tr} \log \Delta)=\exp (\operatorname{Tr} \log (I-\kappa Q)), \\
& \operatorname{Tr} \log (I-\kappa Q)=-\sum_{m} \frac{\kappa^{m}}{m} \operatorname{Tr} Q^{m} \\
&=\sum_{n=-n_{\text {Max }}}^{+n_{\text {Max }}} W_{n} \exp (\mu n / T),(3.5)
\end{aligned}
$$

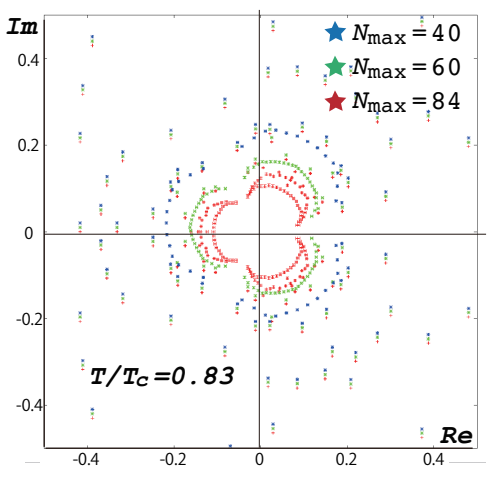

Figure 2: $N_{\max }$ dependence ("Volume" dependence) of Lee-Yang zero distribution. Lattice size is $12^{3} \times 4, \beta=1.5$ and $\kappa=0.1310$. The Lee-Yang zeros are calculated from $Z(\mu, T)=$ $\sum_{n=-N_{\max }}^{+N_{\max }} Z_{n} \xi^{n}$.

here $n$ is called "Winding number". Our first task is to calculate $W_{n}$; Let us examine the inside of Eq. (3.5).

$$
\operatorname{Tr} Q^{m}=\sum_{a, \alpha, x}\left\langle a, \alpha, x\left|Q^{m}\right| a, \alpha, x\right\rangle,
$$

where the trace is taken over the color $(c)$, the spinor $(\alpha)$ and the coordinate $(x)$.

$$
Q^{m}|c, \alpha, x\rangle=\left(\text { CloverTerm }+Q_{i}+e^{+\mu a} Q^{+}+e^{-\mu a} Q^{-}\right)^{m}|c, \alpha, x\rangle=\sum_{k=-m}^{+m} e^{+k \mu a} X(k)^{(m)},
$$

where $a$ is the lattice spacing. Here, $X(k)$ stands for a state vector starting from $(c, \alpha, x)$ and ending at $k$ advanced point along the $t$-direction, where $k$ is positive or negative. See the left panel of Fig 3 , Then,

$$
\left\langle c, \alpha, x\left|Q^{m}\right| c, \alpha, x\right\rangle=\langle c, \alpha, x| \sum_{k} e^{+k \mu a} X(k)^{(m)} .
$$

At the last stage, we pick up the coefficient of $\exp (\mu n / T)$, i.e., $W_{n}$ in Eq 3.5 We describe the process in Algorithm 1.

Now we can calculate the canonical partition functions, $Z_{n}$, via Fourier transformation Eq. 3.3. We observed here numerical instability due to the cancellation of significant digits [8]. We circumvent this by using a multi-precision calculation [25]; we adopt 100 to 200 significant accuracy. 
A difficulty of CA is that $Z_{n}$ become very small when $n$ goes to large, and are fragile against fluctuation.

\subsection{Dark side of the canonical approach}

Using the method, we can calculate the canonical partition functions, $Z_{n}$. Roberge and Weiss pointed out that QCD has the following symmetry [26],

$$
Z\left(\frac{\mu}{T}+i \frac{2 \pi}{N_{c}} k\right)=Z\left(\frac{\mu}{T}\right)
$$

For $N_{c}=3$, using this periodicity, we can write

$$
Z_{n}=\int_{-\pi}^{+\pi} \frac{d \theta}{2 \pi} e^{i n \theta} Z(\theta)=\int_{-\pi}^{+\pi} \frac{d \theta}{2 \pi} e^{i n \theta} \frac{Z(\theta)+Z\left(\theta+\frac{2 \pi}{3}\right)+Z\left(\theta+\frac{4 \pi}{3}\right)}{3}
$$

By setting $\theta^{\prime}=\theta+\frac{2 \pi}{3}$, the second term in Eq. (3.10) is

$$
\int_{-\pi}^{+\pi} \frac{d \theta}{2 \pi} e^{i n \theta} Z\left(\theta+\frac{2 \pi}{3}\right)=\int_{-\pi+\frac{2 \pi}{3}}^{+\pi+\frac{2 \pi}{3}} \frac{d \theta^{\prime}}{2 \pi} e^{i n\left(\theta^{\prime}-\frac{2 \pi}{3}\right)} Z\left(\theta^{\prime}\right)=\int_{-\pi}^{+\pi} \frac{d \theta^{\prime}}{2 \pi} e^{i n \theta^{\prime}} \omega^{2 n} Z\left(\theta^{\prime}\right)
$$

where $\omega \equiv e^{i \frac{2 \pi}{3}}, \quad \omega^{2}=e^{i \frac{4 \pi}{3}}=e^{-i \frac{2 \pi}{3}}$. The third term becomes $\int_{-\pi}^{+\pi} \frac{d \theta^{\prime}}{2 \pi} e^{i n \theta^{\prime}} \omega^{n} Z\left(\theta^{\prime}\right)$. Then

$$
Z_{n}=\int \frac{d \theta}{2 \pi} \frac{1+\omega^{n}+\omega^{2 n}}{3} e^{i n \theta} Z(\theta)
$$

and consequently,

$$
Z_{n}=0, \quad \text { for } \quad n \neq 3 m
$$

We have observed that This "triality" is not satisfied in our simulations. Moreover, $Z_{3 m}$ should be real positive, but this condition is also violated.
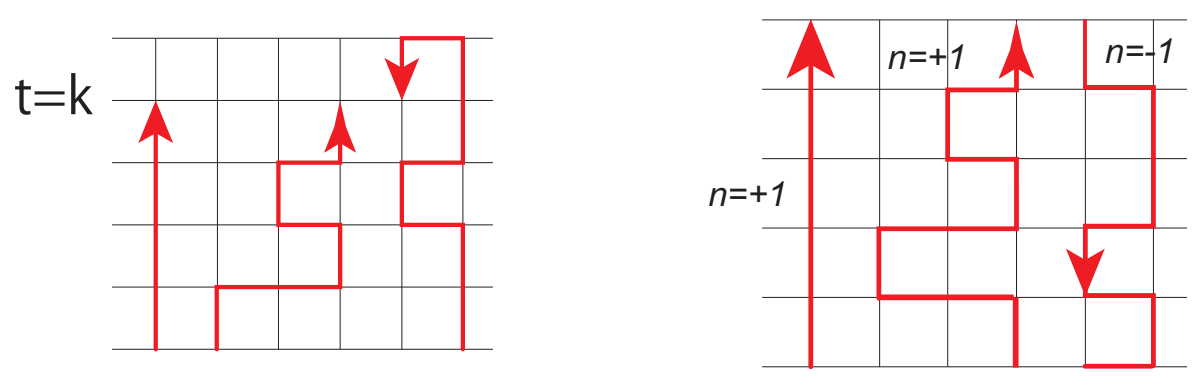

Figure 3: Winding number expansion.

\section{Concluding remarks}

We can beat the "sign problem", in the sense that the complex determinant fades away from the front stage, and newly appearing oscillation of Fourier transformation is under control. And yet now we must fight against an obstacle:

- Triality is not satisfied. 


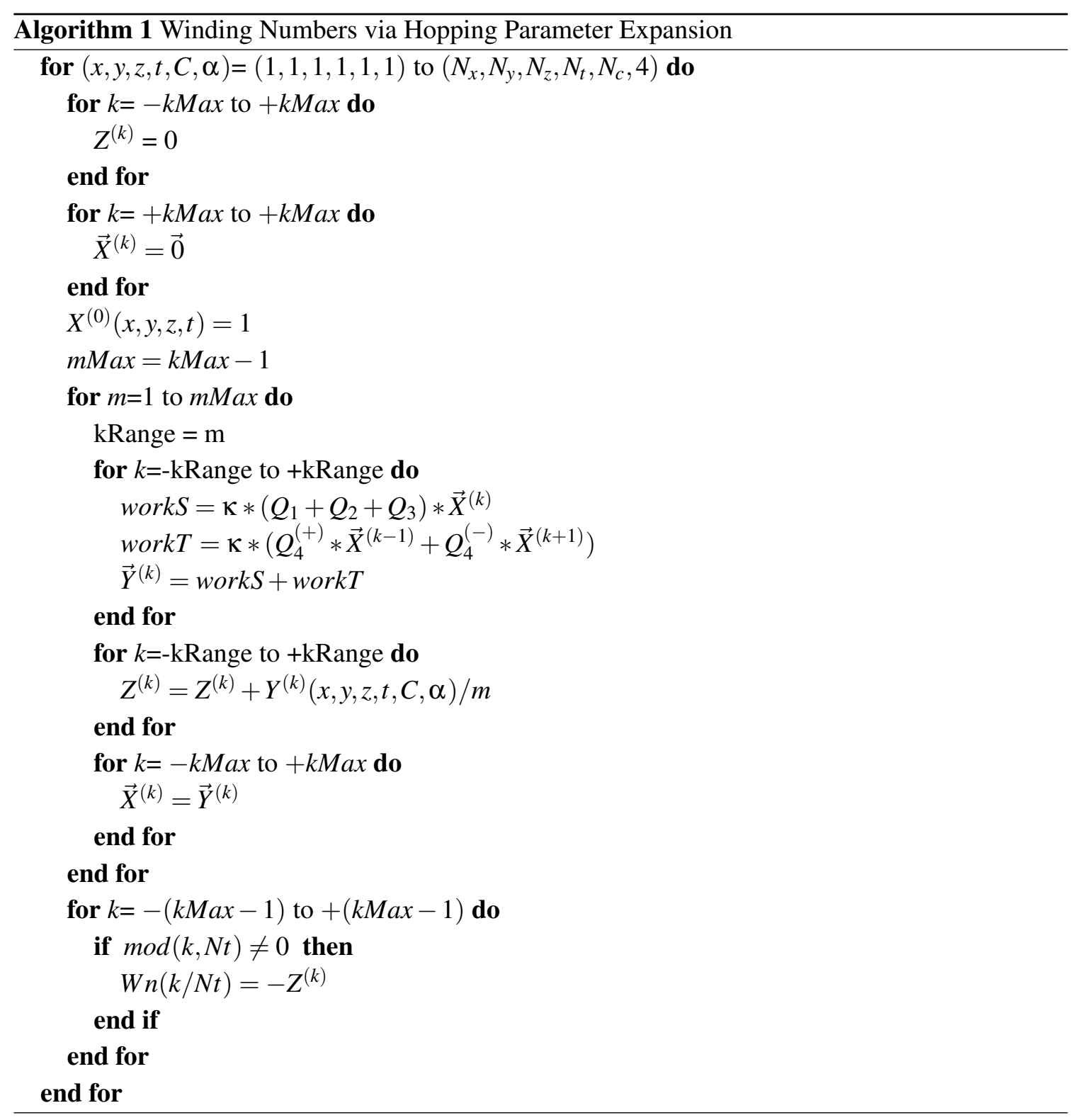

- Canonical partition functions, $Z_{n}$ are not real positive.

This strongly suggests that our Monte Carlo update does not sweep all the configuration space, i.e., the overlap problem.

CA avoid the "sign problem" explicitly. It has advantages of having physically clear meaning, and being able to study not only real chemical potential regions, but also complex chemical potential world. But we should still fight the overlap problem. The triality and real positive $Z_{n}$ are good indicators for that.

\section{Acknowledgement}

We are indebted to A. Alexandru, Ph. de Forcrand and K. Nagata for valuable comments and 
discussions. The calculations were performed on SX-ACE at RCNP Osaka, RICC at Riken and SR16000 at KEK. This work was supported by Grants-in-Aid for Scientific Research $15 \mathrm{H} 03663$ and 26610072, and by the Large Scale Simulation Program No.14/15-19 (FY2014). This work is in part based on Lattice QCD common code Bridge++ [27]. This work is supported by the Large Scale Simulation Program No.14/15-19 (FY2014) of High Energy Accelerator Research Organization (KEK).

\section{References}

[1] A. Nakamura, Phys. Lett. 149B (1984) 391.

[2] D.T. Son, M.A. Stephanov Phys. Rev. Lett. 86 (2001) 592-595

[3] QCD-TARO Collaboration, S. Choe et al., Nucl. Phys. A698, 395 (2002), (hep-lat/0107002)

[4] QCD-TARO Collaboration: I. Pushkina, et al., Phys. Lett. B609 (2005) 265. (hep-lat/0410017).

[5] Y. Taniguchi, to appear in PoS LATTICE 2015.

[6] R. Fukuda, to appear in PoS LATTICE 2015.

[7] A. Suzuki, to appear in PoS LATTICE 2015.

[8] S. Oka to appear in PoS LATTICE 2015.

[9] M. D'Elia, M. P. Lombardo, Proceedings of the GISELDA Meeting held in Frascati, Italy, 14-18 January 2002, hep-lat/0205022.

[10] Ph. de Forcrand, O. Philipsen, Nucl. Phys. B642 290 (2002), hep-lat/0205016.

[11] K. Nagata and A. Nakamura, Phys. Rev. D.83, 114507 (2011) (arXiv:1104.2142).

[12] Z. Fodor, S. D. Katz, JHEP 0203 (2002) 014, (hep-lat/0106002).

[13] K. Nagata and A. Nakamura, JHEP, 1204, 092 (2012). (arXiv:1201.2765)

[14] K. Nagata and A. Nakamura, Phys.Rev. D82 (2010) 094027, [arXiv:1009.2149].

[15] A. Hasenfratz and D. Toussaint, Nucl. Phys. B 371, 539 (1992).

[16] P. de Forcrand and S. Kratochvila, Nucl. Phys. Proc. Suppl. 153, 62 (2006) [hep-lat/0602024].

[17] A. Li, A. Alexandru and K. F. Liu, Phys. Rev. D 84, 071503 (2011) [arXiv:1103.3045 [hep-ph]].

[18] A. Alexandru, M. Faber, I. Horvath and K. F. Liu, Phys. Rev. D 72, 114513 (2005) [hep-lat/0507020].

[19] X. f. Meng, A. Li, A. Alexandru and K. F. Liu, PoS LATTICE 2008, 032 (2008).

[20] A. Li, A. Alexandru, K. F. Liu and X. Meng, Phys. Rev. D 82, 054502 (2010).

[21] J. Danzer and C. Gattringer, Phys. Rev. D 86, 014502 (2012) [arXiv:1204.1020 [hep-lat]].

[22] C. Gattringer and H. P. Schadler, arXiv:1411.5133 [hep-lat].

[23] S. Ejiri, Phys. Rev. D 78, 074507 (2008) [arXiv:0804.3227 [hep-lat]].

[24] C. N. Yang and T. D. Lee, Phys. Rev. 87, 404 (1952), T. D. Lee and C. N. Yang, Phys. Rev. 87 410(1952).

[25] http://myweb.lmu.edu/dmsmith/fmlib.html

[26] A. Roberge and N. Weiss, Nucl. Phys. B275(1986) 734.

[27] http://bridge.kek.jp/Lattice-code/index_e.html 